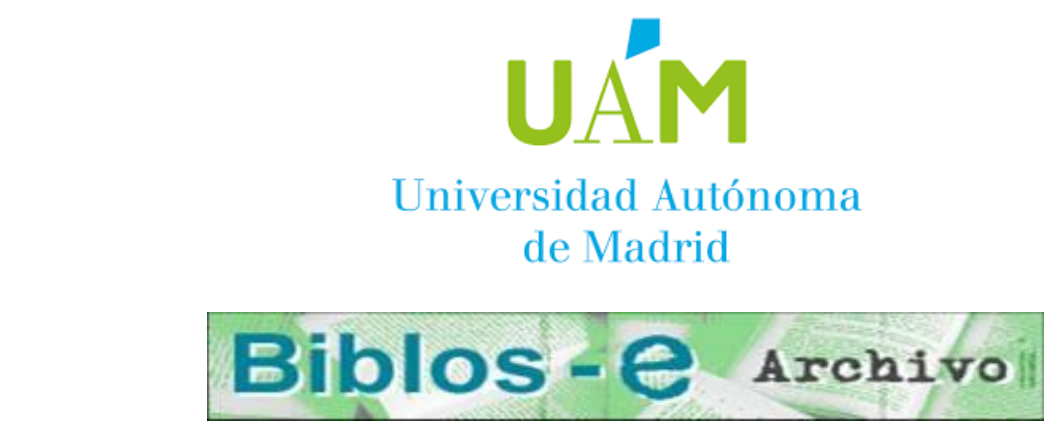

Repositorio Institucional de la Universidad Autónoma de Madrid https://repositorio.uam.es

Esta es la versión de autor del artículo publicado en:

This is an author produced version of a paper published in:

Journal of Materials Chemistry C. 4.36 (2016): 8545-8551

DOI: https://doi.org/10.1039/C6TC02401G

Copyright: (C) The Royal Society of Chemistry 2016

El acceso a la versión del editor puede requerir la suscripción del recurso Access to the published version may require subscription 


\title{
A Crystalline and Free-Standing Silver Thiocarboxylate Thin-Film Showing High Green to Yellow Luminescence
}

\author{
Javier Troyano, ${ }^{a}$ Óscar Castillo, ${ }^{\mathrm{b}}$ Pilar Amo-Ochoa, ${ }^{\mathrm{a}}$ Vanesa Fernández-Moreira, ${ }^{\mathrm{c}}$ Carlos J. Gómez-García, ${ }^{\mathrm{d}}$ Félix Zamora, ${ }^{*, a}$ and \\ Salomé Delgado*,a \\ The simple direct synthesis between $\mathrm{Cu}(\mathrm{II})$ and $\mathrm{Ag}(\mathrm{I})$ salts and thiobenzoic acid under ambient conditions allows the preparation of two bidimensional \\ coordination polymers $[\mathrm{M}(\mathrm{TB})]_{\mathrm{n}}(\mathrm{TB}=$ thiobenzoate; $\mathrm{M}=\mathrm{Cu}(\mathbf{1})$ or $\mathrm{Ag}(\mathbf{2}))$. Their electrical and luminescent properties show that these are multifunctional \\ materials. Interestingly $\mathbf{1}$ and $\mathbf{2}$ undergo a reversible solubilization process. This unusual feature and their simple preparation allow to prepare a \\ crystalline and free-standing thin-film of $\mathbf{2}$, by an interfacial procedure, which shows a remarkable thermochromic luminescence.
}

\section{Introduction}

Thin films are extremely useful towards fabrication of many technological devices including among others smart membranes, catalytic coatings, membranes for selective gas separation and sensors. To this end, several materials have been used going from organic polymers, activated carbons or metal oxides to zeolites and related siliceous materials with defined porous structures. More recently, coordination polymers (CPs) and its porous version metal-organic frameworks (MOFs) has attracted attention in this field. The main reason is based on the modular nature of CPS that allows a molecular design of the final structure and the exceptional physico-chemical properties of this attractive materials that have brought many applications such as catalysts, drug delivery, magnetism, gas storage, and ion exchange..$^{1-9}$

One of the most useful building blocks for the construction of especially eye-catching CPs are organosulfur ligands. These ligands in combination with metal ions give rise to metalorganosulfur networks with interesting electronic properties which are eventually suited for applications in electronics or optoelectronics. ${ }^{10-12}$ In particular, the incorporation of thiolate$S$ as bridging ligands between transition metal sites in CPS seems to produce a synergistic electronic effect since the orbital energies are better matched for sulfur resulting in a

\footnotetext{
a. Departamento de Química Inorgánica and Condensed Matter Physics Center (IFIMAC), Universidad Autónoma de Madrid, E-28049 Madrid, Spain.

b. Departamento de Química Inorgánica, Universidad del País Vasco, Apartado 644, E-48080 Bilbao, Spain.

c. Departamento de Química Inorgánica, Instituto de Síntesis Química y Catálisis Homogénea (ISQCH), CSIC-Universidad de Zaragoza, Zaragoza 50009, Spain. d. Instituto de Ciencia Molecular (ICMol), Universidad de Valencia, 46980 Paterna, Valencia, Spain.

Electronic Supplementary Information (ESI) available: Tables of Crystallographic data, X-ray powder diffractograms, electrical measurements, emission spectra, UV vis, and additional figures. This material is available free of charge via the Internet at http://pubs.acs.org." at DOI: 10.1021/acs.chemater.6b00494. Fig. S1-S15 and Tables S1-S2. See DOI: 10.1039/x0xx00000x
}

greater delocalization of spin density towards the bridging atom. ${ }^{13,14}$

One of the major limitation for the construction of devices based on CPs is their restricted processability. In particular, CP thin films fabrication is still a subject little developed and almost limited to the following procedures. There are three different concepts of MOF thin film fabrication: i) the onsurface direct growth from solvothermal solutions, ii) the assembly of preformed nanocrystals and iii) layer-by-layer growth on the substrate. More recently, few samples have shown the potential of the method based on the CP filmgrowth at the water-air or solvent-solvent interface. This novel approach have produce CP thin-films on substrates and even few of them freestanding.

In this context and following our research interest in the preparation of CPs based on organosulfur ligands and $\mathrm{d}^{10}$ metals ions showing multifunctional properties, ${ }^{15-17}$ this work focuses on the preparation of CPs based on $\mathrm{Cu}(\mathrm{I})$ or $\mathrm{Ag}(\mathrm{I})$ with thiobenzoate ligand, their electrical and optical characterization and their processability as thin-films.

\section{Experimental}

\section{Materials and methods}

All the reagents were purchased from Sigma-Aldrich and used as received. FTIR spectra ( $\mathrm{KBr}$ pellets) were recorded on a Perkin-Elmer 1650 spectrophotometer. C, H, N, S elemental analyses were performed by the Microanalysis Service of the Universidad Autónoma de Madrid on a Perkin-Elmer 240 B microanalyser. Electronic absorption spectra were recorded on an Agilent 8452 diode array spectrophotometer over a range of $\lambda=190-1100 \mathrm{~nm}$ in $0.1,0.2$ and $1 \mathrm{~cm}$ quartz cuvettes thermostated by a Unisoku cryostat. Powder X-ray diffraction experiments were carried out on a Diffractometer PANalyticalX'Pert PRO theta/2theta primary monochromator and detector with fast $X^{\prime}$ Celerator. The samples have been 
analysed with scanning theta/2theta. Direct current (DC) electrical conductivity measurements were performed on different single crystals of compounds $\mathbf{1}$ and $\mathbf{2}$. The contacts were made with wolframium wires ( $25 \mu \mathrm{m}$ diameter) using graphite paste. The samples were measured at $300 \mathrm{~K}$ applying an electrical current with voltages form +10 to $-10 \mathrm{~V}$. Additionally, the thermal dependence of the DC conductivity was measured with the four or two contacts methods (depending on the size of the single crystals) on at least three different single crystals of compounds $\mathbf{1}$ and $\mathbf{2}$ in the temperature range $300-400 \mathrm{~K}$. The contacts were made with $\mathrm{Pt}$ wires $(25 \mu \mathrm{m}$ diameter) using graphite paste. The samples were measured in a Quantum Design PPMS-9 equipment connected to an external voltage source (Keithley model 2400 source-meter) and amperometer (Keithley model 6514 electrometer). The samples were measured by applying a constant voltage of $1 \mathrm{~V}$ and measuring the intensity. All the conductivity quoted values have been measured in the voltage range where the crystals are Ohmic conductors. The cooling and warming rates were $0.5 \mathrm{~K} / \mathrm{min}$ in all cases. Luminescence excitation and emission spectra of the solid compounds were performed at $25^{\circ} \mathrm{C}$ on a 48000 s (T-Optics) spectrofluorometer from SLM-Aminco. A front face sample holder was used for data collection and oriented at $60^{\circ}$ to minimize light scattering from the excitation beam on the cooled R-928 photomultiplier tube. Appropriate filters were used to eliminate Rayleigh and Raman scatters from the emission. Excitation and emission spectra were corrected for the wavelength dependence of the $450 \mathrm{~W}$ xenon arc excitation but not for the wavelength dependence of the detection system. Spectroscopic properties were measured by reflection (front face mode) on finely ground samples and placed in quartz cells of $1 \mathrm{~mm}$ path length. Measurements at variable temperature were done with an Oxford Cryostat Optistat DN.

\section{Synthetic procedures}

Synthesis of $[\mathrm{Cu}(\mathrm{TB})]_{n}(\mathbf{1})$ : A solution of $\mathrm{CuCl}_{2} \cdot 2 \mathrm{H}_{2} \mathrm{O}(0.750 \mathrm{~g}$, $4.35 \mathrm{mmol})$ in ethanol $(20 \mathrm{~mL})$ was added to a solution of HTB (thiobenzoic acid) $(1.400 \mathrm{~g}, 9.12 \mathrm{mmol})$ in ethanol $(20 \mathrm{~mL})$. The mixture was stirred at $20 \stackrel{\circ}{ } \mathrm{C}$ for $2 \mathrm{~h}$ to yield an orange suspension. Then, the precipitate was filtered off, washed with ethanol, chloroform and diethyl ether and finally dried in vacuum. Yield $0.376 \mathrm{mg}$ ( $43 \%$ based on $\mathrm{Cu}$ ). Single crystals of 1 suitable for X-ray diffraction were obtained by slow evaporation at 20 ㅇ $\mathrm{C}$ of a saturated solution of $\mathbf{1}$ in acetonitrile. Anal. Calcd. for $\mathrm{C}_{7} \mathrm{H}_{5} \mathrm{CuOS}$ : $\mathrm{C}, 41.89 ; \mathrm{H}, 2.51$; $\mathrm{S}$, 15.97. Found: $C, 41,65 ; \mathrm{H}, 2.66 ; \mathrm{S}, 15.74$. Selected IR data $(\mathrm{KBr}$ pellet, $\left.\mathrm{cm}^{-1}\right)$ : 3048(w), 1594(m), 1582(s), 1558(s), 1446(m), 1314(w), 1207(s), 1177(m), 1078(w), 1027(w), 919(m), 906(s), 771(s), 687(s), 647(m).

Synthesis of $[\mathrm{Ag}(\mathrm{TB})]_{\mathrm{n}}(2)$ : Following a similar procedure to that for 1 but using $\mathrm{AgNO}_{3}(0.651 \mathrm{~g}, 3.80 \mathrm{mmol}$ ) in ethanol (10 $\mathrm{mL}$ ) and HTB (thiobenzoic acid) $(0.614 \mathrm{~g}, 4.00 \mathrm{mmol}$ ) in ethanol $(10 \mathrm{~mL})$ and wrapping the flask with aluminum foil, a yellow solid was isolated. Yield $0.807 \mathrm{mg}$ ( $87 \%$ based on $\mathrm{Ag}$ ). Single crystals of $\mathbf{2}$ suitable for X-ray diffraction were obtained by diffusion of diethyl ether into a saturated solution of $\mathbf{2}$ in pyridine at 20 으. Anal. Calcd. for $\mathrm{C}_{7} \mathrm{H}_{5} \mathrm{AgOS}$ : $\mathrm{C}, 34.31 ; \mathrm{H}, 2.09$; $\mathrm{S}, 13.06$. Found: $\mathrm{C}, 34.00 ; \mathrm{H}, 2.17 ; \mathrm{S}, 12.96$. Selected IR data (KBr pellet, $\left.\mathrm{cm}^{-1}\right)$ : 2922(w), 1601(s), 1572(s), 1445(m), 1310(w), 1198(s), 1174(m), 1074(w), 1024(w), 917(m), 900(s), 770(s), 684(s), 647(m).

\section{Crystal Structure Determination}

The X-ray diffraction data collections and structure determinations were done at 296(2) K on a Bruker Kappa Apex II diffractometer using graphite-monochromated Mo-Ka radiation ( $\lambda=0.71073 \AA$ ). The cell parameters were determined and refined by a least-squares fit of all reflections. A semiempirical absorption correction (SADABS) was applied for all cases. All the structures were solved by direct methods using the SIR92 program $^{18}$ and refined by full-matrix least-squares on F2 including all reflections (SHELXL97) ${ }^{19}$. All calculations were performed using the WINGX crystallographic software package. ${ }^{20}$ Relevant data acquisition and refinement parameters are gathered in Table S1. CCDC 1481679-1481680 contain the supplementary crystallographic data for this paper.

\section{Results and discussion}

\section{Synthesis and Solution Studies}

The simple one-pot treatment of both $\mathrm{CuCl}_{2}$ or $\mathrm{AgNO}_{3}$ with thiobenzoic acid in ethanol at room temperature leads to the formation of $[M(T B)]_{n}(T B=$ thiobenzoate; $M=C u(1)$ or $A g(2))$. Phase purity was checked with X-ray powder diffraction (Fig. S1 and S2). Note that during the synthesis of 1 the $\mathrm{Cu}(\mathrm{II})$ ions are reduced to $\mathrm{Cu}(\mathrm{I})$ by the action of ethanol, as already observed in other cases.

Recently, we have observed that $\mathrm{CPs}$ based on $\mathrm{Cu}(\mathrm{I})$ or $\mathrm{Ag}(\mathrm{I})$ with thioacetamide show relatively high solubility in several solvents giving rise to reversible systems between the starting CPs and the solubilized building blocks present in solution. ${ }^{17}$ These observations prompted us to carry similar studies with $\mathbf{1}$ and 2. Thus, we observed that testing several solvents (water, methanol, ethanol, dichlorometane, tetrahydrofuran, toluene, $\mathrm{N}, \mathrm{N}^{\prime}$-dimethylformamide and acetone) $\mathbf{1}$ is only sparingly soluble in acetonitrile whereas $\mathbf{2}$ highly solubility in pyridine. Indeed, solutions of $\mathbf{1}$ in acetonitrile were followed by UV-vis spectroscopy, leading to a spectrum where the absorption bands at 227 and $311 \mathrm{~nm}$, corresponding to thiobenzoate anion, were observed (Fig. S3). The presence of this anion is clearly identified by comparison with the registered spectrum of the ammonium thiobenzoate generated in situ under the same conditions. We have also verified the reversibility of this process. Thus, a saturated acetonitrile solution of 1 reverts to the starting compound by controlled elimination of the solvent, allowing its recrystallization (characterized by CNHS analyses, IR spectroscopy and X-ray powder diffraction) (Fig. S4). Similar studies were carried out for 2, however this material was only readily soluble in pyridine. Pyridine solution of $\mathbf{2}$ also reverts to the initial crystalline polymer when the solvent was eliminated (characterized by CNHS analyses, IR 
spectroscopy and X-ray powder diffraction), confirming the reversibility of this process (Fig. S5).

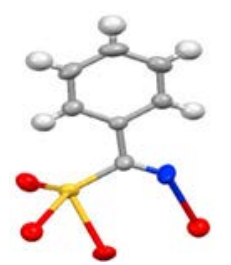

(a)

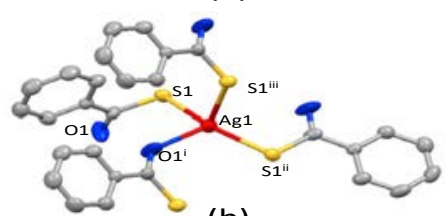

(b)

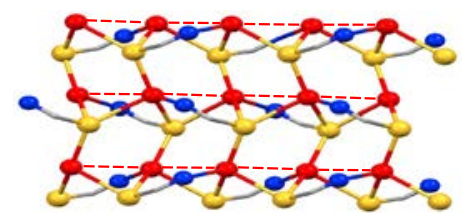

(c)

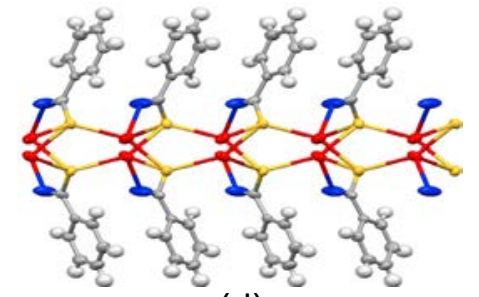

(d)

Fig. 1 Coordination mode of the thiocarboxylate ligand (a), coordination environment (b), fragment of the polymeric 2D sheet in which the phenyl rings of the thiocarboxylato ligands have been omitted for clarity (c), and lateral view of the $2 \mathrm{D}$ coordination polymer (d) of compound 2. Dashed lines indicate Ag ‥Ag contacts.

\section{Crystal Structures}

Compound $\mathbf{2}$ is isostructural to compound $\mathbf{1}$, previously described by Bhattacharya et al. ${ }^{21}$ Its crystal structure is comprised of a two-dimensional coordination network in which the $\mathrm{Ag}^{+}$center is placed on a distorted tetrahedral environment surrounded by three sulfur and one oxygen atoms of four different thiobenzoate moieties (Fig. 1). The benzoate ligand exhibits a quite exotic coordination mode in which the sulfur atom is coordinated to three silver(I) centers whereas the oxygen atom is coordinated to only one. The silver atom is placed at the center of four six-membered fused rings $\left(\mathrm{Ag}_{3} \mathrm{~S}_{3}\right.$ and $\left.\mathrm{Ag}_{2} \mathrm{COS}_{2}\right)$, which are in a boat (or twisted boat) conformation. This coordination mode generates a bidimensional planar coordination polymer with the phenyl decorating both sides of the planar sheet and a width of 1.6 $\mathrm{nm}$. Topological analysis shows a $10-\mathrm{c}$ uninodal net with point symbol $\left\{3^{21} \cdot 4^{21} \cdot 5^{3}\right\}$. The layers are held together by means of weak van der Waals interactions. Apart from the expected lengthening of the coordination bond distances because of the bigger size of $\mathrm{Ag}^{+}$compared to $\mathrm{Cu}^{+}$, the main difference with respect to compound $\mathbf{1}$ comes from the metal..mmetal distances that are above the sum of the van der Waals radii for copper ( 3.17 vs $2.80 \AA$ ) in compound 1 but significantly below it for the case of silver...silver distances in compound 2 (3.07 vs $3.44 \AA)$.

Table S2 gathers the most relevant structural parameters for this compound. This fact becomes more evident as the temperature lowers. The crystal structure at $100 \mathrm{~K}$ shows the same structural features as those described above but the usual slight shrinkage of the bond distances with the lowering of the temperature also affects the metal $\cdots$ metal distances which become even shorter (3.04 $\AA$ ). Previous studies have shown that these small changes on metal...metal distances can play a key role on the electrical and optical behavior of these semiconducting materials. ${ }^{16,22-27}$

\section{Electrical conductivity}

Coordination polymers of $\mathrm{Cu}(\mathrm{I})$ centers bridged by sulfurcontaining ligands seems to be especially suitable to show electrical conductivity due to the excellent metal-sulfur orbital overlapping. ${ }^{11}$ Accordingly, we have measured IV characteristics of compounds $\mathbf{1}$ and $\mathbf{2}$ using direct current (DC) at $300 \mathrm{~K}$ in several single crystals (Fig. S6 and S7). The almost Ohmic curves obtained show average DC conductivity values at $300 \mathrm{~K}$ of $1.3 \times 10^{-6}$ and $1.1 \times 10^{-7} \mathrm{~S} \mathrm{~cm}^{-1}$ for 1 and 2 , respectively, suggesting a semiconductor behavior (Table 1 ). In order to confirm it, we have also performed DC electrical conductivity measurements with the four contacts method as a function of the temperature for compounds $\mathbf{1}$ and $\mathbf{2}$ (Fig. S8). These measurements show that both compounds are semiconductors (i.e. the resistivity decreases with decreasing the temperature) and follow a classical Arrhenius-type semiconducting behavior with activation energies of 0.64 and $1.02 \mathrm{eV}$ in $\mathbf{1}$ and 2, respectively.

Table 1 Electrical conductivity data obtained at 300K for 1 and 2 using two contact method and Metal-Sulphur distances (M-S).

\begin{tabular}{cccc}
\hline Comp. & $\sigma_{300 \mathrm{~K}}\left(\mathrm{~S} \mathrm{~cm}^{-1}\right)$ & $\mathrm{E}_{\mathrm{a}}(\mathrm{eV})$ & $\mathrm{M}-\mathrm{S}$ distance $(\AA)$ \\
\hline $\mathbf{2}$ & $1.3 \times 10^{-6}$ & 0.64 & $2.280-2.492$ \\
\hline
\end{tabular}

Despite the fact that a metal-metal zig-zag linear chain extended in the [100] direction along the CP layers shows metal-to-metal distances of $3.179 \AA$ and $3.070 \AA$ for 1 and 2, respectively. While this $\mathrm{Cu}-\mathrm{Cu}$ distance is $0.379 \AA$ larger than the sum of the van der Waals radii, the Ag-Ag distance is 0.370 $\AA$ below. Therefore confirming stronger metal-metal interactions in $\mathbf{2}$ than in 1. However, in these isostructural compounds we observe that metal-metal distances cannot explain both the lower activation energy and the higher conductivity found for $\mathbf{1}$. We suggest that the current rather than traveling along the metal-metal bonds are mediated by the extended metal-sulfur (M-S) bonds. These M-S distances fall in the range of 2.280-2.492 $\AA$ for 1 , and 2.464-2.890 $\AA$ for 2. Comparing the structural features of $\mathbf{1}$ and $\mathbf{2}$, the larger $\mathrm{M}-\mathrm{S}$ distances are those found for $\mathbf{2}$, in agreement with the lower 
conductivity value and with the higher activation energy. The suggested mechanism is in agreement to that found in similar $\mathrm{CP}$ containing organosulfur ligands such as $(\mathrm{MXTAA})_{n},(\mathrm{M}=\mathrm{Cu}$, $\mathrm{Ag}, \mathrm{X}=\mathrm{Br}, \mathrm{I}$; TAA = thioacetamide), ${ }^{38}$ in which conductivity values are related to the metal-sulfur distances.

\section{Luminescent properties}

CPs have recently attracted attention due to their luminescent properties and potential applications as light emitting diodes. ${ }^{28,29,30}$ The arrangement of the metal centers and the electronic structure of the molecules in the excited state have important influence in the photoluminescence. Thus CP based on metal ions with $\mathrm{d}^{10}$ electronic configuration have become attractive as luminescent materials. ${ }^{31,32}$ In this context, $\mathrm{Cu}(\mathrm{I})$ complexes are particularly suited because they show rich structural variety and bright luminescent even at room temperature. ${ }^{33}$ However the $\mathrm{Ag}(\mathrm{I})$ analogues are typically nonemissive at room temperature or exhibit weak photoluminescence centered at around $480 \mathrm{~nm} \cdot{ }^{34,35}$ The luminescent properties of $\mathbf{1}$ and $\mathbf{2}$ were investigated in the solid state. Thus, at room temperature, excitation of solid samples at $\lambda=359 \mathrm{~nm}$ (Fig. S9) produces weak emission for 1, with two peaks centered at 438 and 493 (br) nm, and a very weak emission centered at $612 \mathrm{~nm}$, while 2 yields more intense green photoluminescence with emission peaks at 439 , 496 (sh) and $510 \mathrm{~nm}$. A feasible assignment of the excited states of $d^{10}$ complexes to ligand-centered $\pi \rightarrow \pi^{*}$ transitions (LC), ligand-to-ligand (LLCT), ligand-to-metal (LMCT), or metalto-ligand (MLCT) charge-transfer transitions or metal-centered $d^{10} \rightarrow d^{9} s^{1}$ (MCC) transitions can explain these emission peaks. ${ }^{36}$ For both compounds the emission peaks observed at higher energies are essentially the same to those found in solid-state fluorescence of free thiobenzoic acid (Fig. S10).

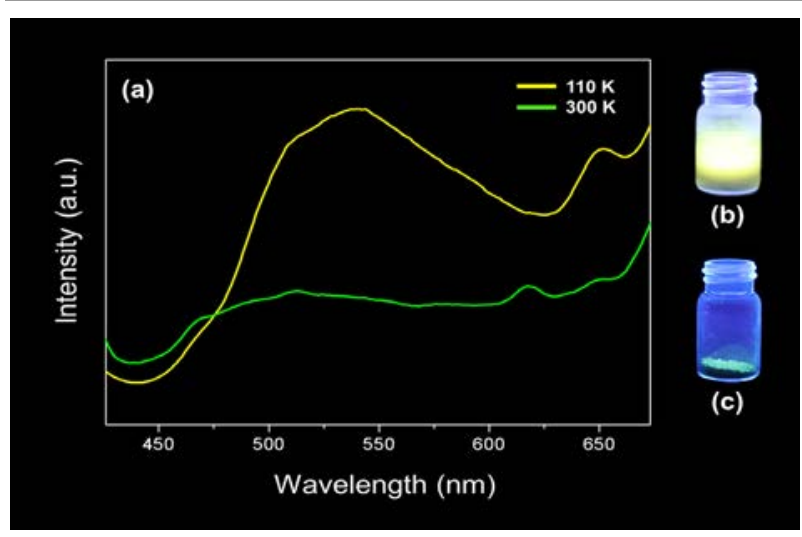

Fig. 2 (a) Photoluminescence spectra for 2 at different temperatures $\left(\lambda_{\mathrm{ex}}=375 \mathrm{~nm}\right)$ in the solid-state. Photographs of a glass vial containing solid sample of 2 at (b) 77 and (c) $298 \mathrm{~K}$ irradiated with a UV-lamp $\left(\lambda_{\mathrm{ex}}=365 \mathrm{~nm}\right)$.

We believe that these peaks observed in the same region for $\mathbf{1}$ and $\mathbf{2}$ are due to ligand-centered $\pi \rightarrow \pi^{*}$ transitions and/or intraligand transitions processes. Additionally, 2 shows a low energy emission band centered at $510 \mathrm{~nm}$. This band can be associated to the ligand to-metal charge-transfer LMCT excited state, that is charge transfer from the $\mathrm{S} 3 \mathrm{p}$ to $\mathrm{Ag} 5 \mathrm{~s}$ orbitals, perturbed by $\mathrm{Ag} \cdots \mathrm{Ag}$ interactions, ${ }^{37}$ which is characteristic of the polynuclear $d^{10}$ complexes with metallophilic interactions. ${ }^{38}$

Interestingly, when $\mathbf{2}$ was packed into a tube and then immersed in liquid nitrogen for a few minutes and exposed to the irradiation of the UV lamp, changes to intense yellow emission can be easily distinguished by naked eye that reversible shift to the initial green upon warming to room temperature, indicating a fully reversible thermochromic luminescence process. To investigate the effects of measurement temperature, the emission spectra of $\mathbf{2}$ in the solid state was recorded from 300 to $110 \mathrm{~K}$, showing interesting luminescence thermochromism (Fig. 2). Thus, at $110 \mathrm{~K}$ a red shift of $32 \mathrm{~nm}\left(\lambda_{\max }=542 \mathrm{~nm}\right)$ is observed with respect to the emission band at $300 \mathrm{~K}$. Additionally, with the decrease of temperature, an increment of the intensity of emission band was detected. The temperature dependent luminescence colors of $\mathbf{2}$ from green to yellow on moving from 298 to $110 \mathrm{~K}$, can be clearly observed by the naked eye (Fig. 2b and $2 \mathrm{c}$ ). The exhibited luminescence thermochromism is rather uncommon because although there are some reports on luminescence thermochromism on silver thiolate compounds, ${ }^{39}$ those are just emissive in the solid state at low temperature but non-emissive at room temperature. ${ }^{40}$

For a better understanding of this phenomenon single-crystal diffraction analyses on compound $\mathbf{2}$ at $110 \mathrm{~K}$ were carried out reveling that no phase transition occurs upon cooling but some structural changes. The detailed crystal data and structure refinement and selected bond lengths of $\mathbf{2}$ at this temperature are given in Table S1 and S2. Obvious shrinkage of unit-cell size from $1417.2(6) \AA^{3}(298 \mathrm{~K})$ to $1393.2(6) \AA^{3}$ was observed, which is accompanied by shortening of $\mathrm{Ag} \cdots \mathrm{Ag}$ bond distances, from $3.0701(2) \AA(298 \mathrm{~K})$ to $3.044(2) \AA(110 \mathrm{~K})$, much shorter than the sum of the van der Waals radii $(3.44 \AA) .{ }^{41}$ Therefore, the shift observed to lower energy $(\Delta=32 \mathrm{~nm})$ when the temperature decreases is probably associated with stronger argentophilic interactions and the higher intensity of emission at lower temperature can be due to a higher structural rigidity in the compound which reduces the non-radiative decay of the emitting excited state. ${ }^{40}$

\section{Thin-Film Preparation and Characterization}

It is well known that CPs have limited solubility or no solubility in common organic solvents, hampering their solution processability. However, few CPs show the ability to reversible dissociate under specific conditions in solution. ${ }^{17}$

Therefore, taking into account the high solubility in pyridine exhibited by $\mathbf{2}$, and because of its interesting luminescent properties, we decided to explore its processabilty. Our first strategy was to confine the polymer recrystallization at the airwater interface. Thus, $10 \mu \mathrm{L}$ of a pyridine solution of 2 (10 g $\mathrm{L}^{-1}$ ) were carefully added on a water surface. Immediately after a film was formed at the air-water interface. Then, the film was transferred to a glass substrate by Langmuir Schaefer (LS) method $^{42}$ and dried with an argon flow. Grazing incident X-ray diffraction (GIXRD) confirmed that the structure of the 
film consist of that observed for $\mathbf{2}$ (Fig. S11a). However, fieldemission scanning electron microscopy (FE-SEM) study showed that the surface has non-homogeneous coverage but consist of micron-size disks formed by uniformly distributed aggregates of nanoparticles of diameter close to 50-100 nm (Fig. S11b-d). AFM allows to estimate a thickness of ca. 5-10 nm (Fig. S11e,f). In order to obtain a continuous film of $\mathbf{2}$, we tried the liquidliquid interfacial synthesis method. In this approach, crystallization takes place at the liquid-liquid interface, where the reagents come into contact through a diffusion phenomenon. ${ }^{43-47}$ Thus we used a solution of thiobenzoic acid $(0.015 \mathrm{~g}, 0.10 \mathrm{mmol})$ in $n$-pentane $(5 \mathrm{~mL})$ that was added to the surface of a solution of $\mathrm{AgNO}_{3}(0.017 \mathrm{~g}, 0.10 \mathrm{mmol})$ in distilled water $(10 \mathrm{~mL})$. After $c a .10$ minutes film formation at the water-pentane interface was observed with naked eyes (Fig. 3a). Evaporation of $n$-pentane at 20 ㅇ C (about half an hour) produce the isolation of the film on the top of the water. Then the film was deposited on several substrates by LS method and dried with an argon flow. Optical inspection of the surface showed a homogeneous coverage with millimetric areas (Fig. S12). Concentration appears to be a key factor in achieving good coverings, since at lower concentrations a nonhomogeneous covering of the surface was observed (Fig. S13). The powder-XRD pattern of these films was in good agreement with that show for $\mathbf{2}$ (Fig. 3b). FE-SEM images showed detailed morphology of the typical products obtained (Fig. 3c-e). The films consist of the aggregate of nano-sized particles, with a size range from 100 to $200 \mathrm{~nm}$. Interestingly Fig. 3c and $3 f$ show free-standing films. Fig. $3 f$ displays the cross-section, where the thickness is estimated to be $c a .1 \mu \mathrm{m}$.

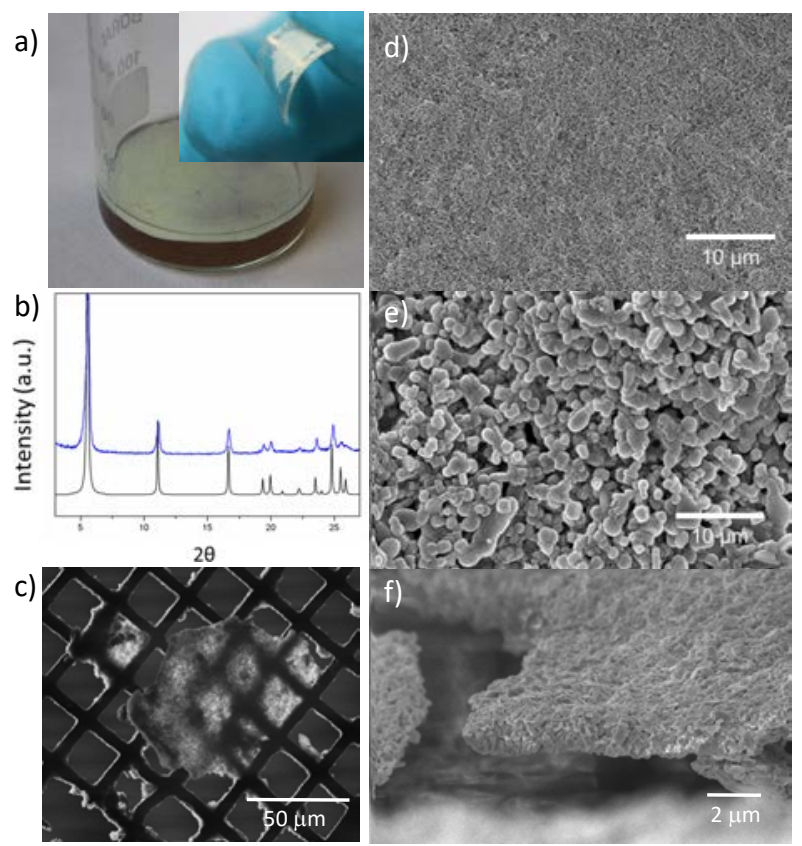

Fig. 3 a) Photograph of $\mathbf{2}$ film generated at the interface between aqueous solution and pentane. The inset shows film transferred on PDMS flexible substrate. b) XRPD pattern of the film obtained by liquid-liquid interfacial synthesis (blue) and simulated XRPD pattern of $\mathbf{2}$ (black). c-f) FE-SEM images at different magnifications showing: c) a freestanding film deposited on a copper TEM grid, d) a low magnification view of the film, e) a zoomed area showing the aggregation of nanoparticles and f) a cross-section of a free-standing thin film.
During the transfer process we observed that the films of $\mathbf{2}$ can be handled as free-standing thin films, due to their intrinsic mechanical strength, as can be seen in Fig. S14. Additionally, thin films of $\mathbf{2}$ can also be deposited on flexible substrate, such as poly(dimethylsiloxane) (PDMS) (Fig. S15). This would open the door to future applications in flexible optical displays.

Finally, we observed that the exceptional emitting features of 2 are also present in the thin-film. Thus, Fig. 4 shows the emission of $\mathbf{2}$ film deposited on a glass substrate under irradiation at $365 \mathrm{~nm}$ at 77 and $298 \mathrm{~K}$ showing the green and yellow emission observed in bulk.

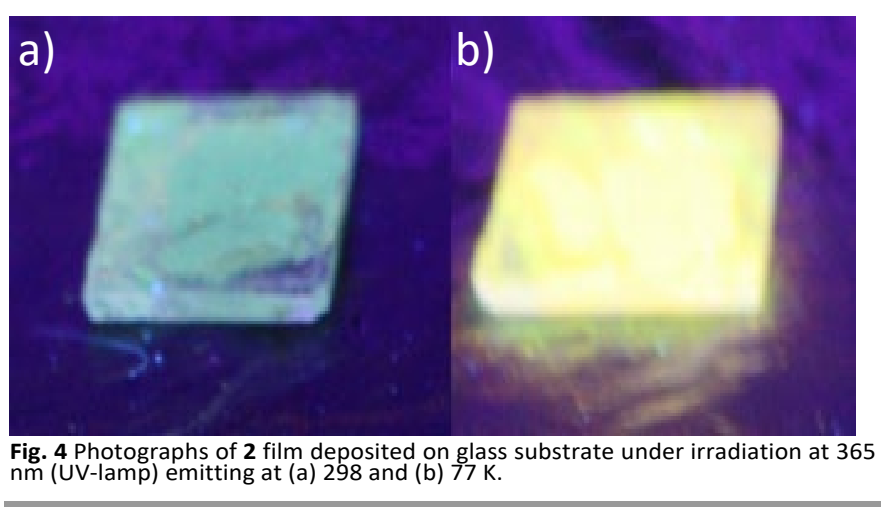

\section{Conclusion}

Two coordination polymers with $\mathrm{Cu}(\mathrm{I})$ or $\mathrm{Ag}(\mathrm{I})$ and thiobenzoate ligand have been prepared under ambient conditions, $[M(T B)]_{n}(T B=$ thiobenzoate; $M=C u(1)$ or $A g(2))$. These materials are semiconductors and show emission that is very strong in the case of the silver derivative. This is very unusual since typically silver CPs are poor emitters. However, $\mathbf{2}$ shows a thermochromic behavior from green to yellow emission upon cooling from 298 to $77 \mathrm{~K}$. Interestingly, 1 and $\mathbf{2}$ can be dissolved in acetonitrile and pyridine, respectively, to form solutions containing the initial building blocks. These solutions reverse to the formation of $\mathbf{1}$ and $\mathbf{2}$ upon solvent evaporation.

The two exceptional chemical features of these CPs, simple and soft reaction condition formation and reversibility in solution with certain solvents, allow us to test different procedures to form thin-films. Thus a simple interfacial procedure has allowed the preparation of a highly crystalline and free-standing thin-film of $\mathbf{2}$ that shows a remarkable thermochromic luminescence.

\section{Acknowledgements}

This work was supported by MICINN (MAT2013-46753-C2-1-P and reference CTQ2013-48635-C2-1-P) and Generalitat Valenciana (projects Prometeoll/2014/076 and ISIC). Authors are indebted with Prof. Concepción Gimeno (Universidad de Zaragoza-CSIC, Spain) for their assistance in luminescence measurements. 


\section{Notes and references}

1 S. Kitagawa and S. Noro, Comprehensive Coordination Chemistry, 2004.

2 B. Moulton and M. J. Zaworotko, Chem. Rev., 2001, 101, 1629-1658.

3 N. Stock and S. Biswas, Chem. Rev., 2012, 112, 933-969.

4 S. T. Meek, J. A. Greathouse and M. D. Allendorf, Adv. Mater., 2011, 23, 249-267.

5 W. L. Leong and J. J. Vittal, Chem. Rev., 2011, 111, 688-764.

6 C. Janiak and J. K. Vieth, New J. Chem., 2010, 34, 2366-2388.

7 D. J. Tranchemontagne, J. L. Mendoza-Cortes, M. O'Keeffe and O. M. Yaghi, Chem. Soc. Rev., 2009, 38, 1257-1283.

8 M. Hong, Cryst. Growth Des., 2007, 7, 10-14.

9 S. Diring, D. O. Wang, C. Kim, M. Kondo, Y. Chen, S. Kitagawa, K. Kamei and S. Furukawa, Nat. Commun., 2013, 4, Art. Num. 2684.

10 H.-B. Zhu and S.-H. Gou, Coord. Chem. Rev., 2011, 255, 318338.

11 G. Givaja, P. Amo-Ochoa, C. J. Gomez-Garcia and F. Zamora, Chem. Soc. Rev., 2012, 41, 115-147.

12 J. A. García-Vázquez, J. Romero and A. Sousa, Coord. Chem. Rev., 1999, 193-195, 691-745.

13 S. S. Alexandre, J. M. Soler, P. J. Sanz Miguel, R. W. Nunes, F. Yndurain, J. Gómez-Herrero and F. Zamora, Appl. Phys. Lett., 2007, 90, 193107

14 D. Tiana, C. H. Hendon, A. Walsh and T. P. Vaid, Phys. Chem Chem. Phys., 2014, 16, 14463-14472.

15 S. Delgado, P. J. Sanz Miguel, J. L. Priego, R. Jiménez-Aparicio, C. J. Gómez-García and F. Zamora, Inorg. Chem., 2008, 47, 9128-9130.

16 A. Gallego, O. Castillo, C. J. Gomez-Garcia, F. Zamora and S. Delgado, Inorg. Chem., 2012, 51, 718-727.

17 J. Troyano, J. Perles, P. Amo-Ochoa, J. I. Martinez, F. Zamora and S. Delgado, CrystEngComm, 2014, 16, 8224-8231.

18 A. Altomare, G. Cascarano, C. Giacovazzo and A. Guagliardi, J. Appl. Crystallogr., 1993, 26, 343-350.

19 G. M. Sheldrick, SHELXL-97, Program for Crystal Structure Refinement, 1997.

20 L. Farrugia, J. Appl. Crystallogr., 1999, 32, 837-838.

21 D. K. Joshi, K. B. Mishra, V. K. Tiwari and S. Bhattacharya, RSC Adv., 2014, 4, 39790-39797.

22 S. Delgado, A. Gallego, O. Castillo and F. Zamora, Dalton Trans., 2011, 40, 847-852.

23 S. Delgado, A. Santana, O. Castillo and F. Zamora, Dalton Trans., 2010, 39, 2280-2287.

24 A. Gallego, O. Castillo, C. J. Gomez-Garcia, F. Zamora and S. Delgado, Eur. J. Inorg. Chem., 2014, 3879-3887.

25 A. Gallego, C. Hermosa, O. Castillo, I. Berlanga, C. J. GomezGarcia, E. Mateo-Marti, J. I. Martinez, F. Flores, C. GomezNavarro, J. Gomez-Herrero, S. Delgado and F. Zamora, Adv. Mater., 2013, 25, 2141-2146.

26 A. I. Gallego, O. Castillo, F. Zamora and S. Delgado, RSC Adv., 2013, 3, 18406-18413.

27 M. L. Gallego, A. Guijarro, O. Castillo, T. Parella, R. MasBalleste and F. Zamora, CrystEngComm, 2010, 12, 2332 2334.

28 E. Cariati, X. H. Bu and P. C. Ford, Chem. Mater., 2000, 12, 3385-3391.

29 D. M. Ciurtin, N. G. Pschirer, M. D. Smith, U. H. F. Bunz and H. C. zur Loye, Chem. Mater., 2001, 13, 2743-2745.

30 F. Wurthner and A. Sautter, Chem. Commun., 2000, 445-446.

31 H. A. Habib, A. Hoffmann, H. A. Hoeppe, G. Steinfeld and C. Janiak, Inorg. Chem., 2009, 48, 2166-2180.

32 V. W.-W. Yam and K. M.-C. Wong, Chem. Commun., 2011, 47, 11579-11592.

33 P. C. Ford, E. Cariati and J. Bourassa, Chem. Rev., 1999, 99, 3625-3648
34 C. Janiak, Dalton Trans., 2003, 2781-2804.

35 Y.-B. Dong, Q. Zhang, L. Wang, J.-P. Ma, R.-Q. Huang, D.-Z. Shen and D.-Z. Chen, Inorg. Chem., 2005, 44, 6591-6608.

36 V. Wing-Wah Yam and K. Kam-Wing Lo, Chem. Soc. Rev., 1999, 28, 323-334.

37 A. Barbieri, G. Accorsi and N. Armaroli, Chem. Commun., 2008, 2185-2193.

38 V. W.-W. Yam, Acc. Chem. Res., 2002, 35, 555-563.

39 S. Yuan, Y.-K. Deng, X.-P. Wang and D. Sun, New J. Chem., 2013, 37, 2973-2977.

40 D. Sun, H. Wang, H.-F. Lu, S.-Y. Feng, Z.-W. Zhang, G.-X. Sun and D.-F. Sun, Dalton Trans., 2013, 42, 6281-6284.

41 A. Bondi, J. Phys. Chem., 1964, 68, 441-451.

42 I. Langmuir and V. J. Schaefer, J. Am. Chem. Soc., 1938, 60, 1351-1360.

43 H. Lu and S. Zhu, Eur. J. Inorg. Chem., 2013, 2013, 1294-1300.

44 R. Ameloot, F. Vermoortele, W. Vanhove, M. B. J. Roeffaers, B. F. Sels and D. E. De Vos, Nat. Chem., 2011, 3, 382-387.

45 T. Pal, T. Kambe, T. Kusamoto, M. L. Foo, R. Matsuoka, R. Sakamoto and H. Nishihara, ChemPlusChem, 2015, 80, 12551258.

46 T. Kambe, R. Sakamoto, K. Hoshiko, K. Takada, M. Miyachi, J.H. Ryu, S. Sasaki, J. Kim, K. Nakazato, M. Takata and H. Nishihara, J. Am. Chem. Soc., 2013, 135, 2462-2465.

47 X. Huang, P. Sheng, Z. Tu, F. Zhang, J. Wang, H. Geng, Y. Zou, C.-a. Di, Y. Yi, Y. Sun, W. Xu and D. Zhu, Nat. Commun., 2015, 6, Art. Num. 7408. 